\title{
The Topological Classification of Diffeomorphisms of the Two-Dimensional Torus with an Orientable Attractor
}

\author{
V.Z. Grines, E. V. Kruglov, O. V. Pochinka
}

This paper is devoted to the topological classification of structurally stable diffeomorphisms of the two-dimensional torus whose nonwandering set consists of an orientable one-dimensional attractor and finitely many isolated source and saddle periodic points, under the assumption that the closure of the union of the stable manifolds of isolated periodic points consists of simple pairwise nonintersecting arcs. The classification of one-dimensional basis sets on surfaces has been exhaustively obtained in papers by V. Grines. He also obtained a classification of some classes of structurally stable diffeomorphisms of surfaces using combined algebra-geometric invariants. In this paper, we distinguish a class of diffeomorphisms that admit purely algebraic differentiating invariants.

Keywords: A-diffeomorphisms of a torus, topological classification, orientable attractor

This work was performed with support of the Laboratory of Dynamical Systems and Applications NRU HSE of the Ministry of science and higher education of the RF grant no 075-15-2019-1931.

Vyacheslav Z. Grines

vgrines@yandex.ru

Olga V. Pochinka

olga-pochinka@yandex.ru

National Research University Higher School of Economics

ul. B. Pecherskaya 25/12, Nizhny Novgorod, 603150 Russia

Evgeniy V. Kruglov

kruglov19@mail.ru

Lobachevsky State University of Nizhny Novgorod

prosp. Gagarina 23, Nizhny Novgorod, 603950 Russia 


\section{Introduction and main results}

In this paper we study structurally stable diffeomorphisms $f$ defined on the two-dimensional torus $\mathbb{T}^{2}$ and containing a one-dimensional orientable attractor $\Lambda$ in the nonwandering set $N W(f)$. It follows from the results of V. Grines [4, 6] (see also the monograph [5]) that in this case

- $N W(f)$ does not contain orientable basic sets other than $\Lambda$,

- the basic set $\Lambda$ has only bunches of degree two that divide the boundary points into associated pairs $p_{i}, q_{i}, i \in\{1, \ldots, k\}$,

- the induced isomorphism $f_{*}: \pi_{1}\left(\mathbb{T}^{2}\right) \rightarrow \pi_{1}\left(\mathbb{T}^{2}\right)$ is uniquely defined by the hyperbolic matrix $A_{f} \in G L(2, \mathbb{Z})$.

We will denote by $\widehat{A}: \mathbb{T}^{2} \rightarrow \mathbb{T}^{2}$ the diffeomorphism given by the formula

$$
\widehat{A}(x, y)=(\alpha x+\beta y, \gamma x+\delta y) \quad(\bmod 1)
$$

for any matrix $A=\left(\begin{array}{ll}\alpha & \beta \\ \gamma & \delta\end{array}\right) \in G L(2, \mathbb{Z})$.

According to J. Franks [1], there is a unique, isotopic to the identity, continuous map $h_{f}: \mathbb{T}^{2} \rightarrow \mathbb{T}^{2}$ that semiconjugates the diffeomorphism $f$ with the diffeomorphism $\widehat{A}_{f}$. The image $h_{f}(\Lambda)$ of the set $\Lambda$ is the whole torus $\mathbb{T}^{2}$, and the set $B_{f}=\left\{x \in \mathbb{T}^{2}: h_{f}^{-1}(x)\right.$ consisting of more than one point $\}$ is the union of finitely many periodic points $P_{f}=\left\{\varrho_{1}, \varrho_{2}, \ldots, \varrho_{k}\right\}$ of the diffeomorphisms $\widehat{A}_{f}$ and their unstable manifolds. In this case, $h_{f}^{-1}\left(\varrho_{i}\right) \cap \Lambda, i \in\{1,2, \ldots, k\}$, consists of a pair of associated boundary points $p_{i}, q_{i}$ of the basic set $\Lambda$.

Denote by $G$ the class of structurally stable diffeomorphisms $f: \mathbb{T}^{2} \rightarrow \mathbb{T}^{2}$ such that $N W(f)$ consists of an orientable one-dimensional attractor $\Lambda$ and a finite number of isolated periodic points whose stable manifolds closure belongs to simple arcs $L_{p_{i} q_{i}}$ bounded by pairs of boundary points $p_{i}, q_{i}$ (see Fig. 1). Then $h_{f}\left(L_{p_{i} q_{i}}\right)=\varrho_{i}$. Denote by $n_{\varrho_{i}}$ the number of sources on the $\operatorname{arc} L_{p_{i} q_{i}}$.

The following theorem is the main result of this paper.

Theorem 1. The diffeomorphisms $f, f^{\prime} \in G$ are topologically conjugate if and only if there exists a matrix $H \in G L(2, \mathbb{Z})$ such that $H A_{f}=A_{f^{\prime}} H, \widehat{H}\left(P_{f}\right)=P_{f^{\prime}}$ and $n_{\varrho_{i}}=n_{\widehat{H}\left(\varrho_{i}\right)}$, $i=1, \ldots, k$.

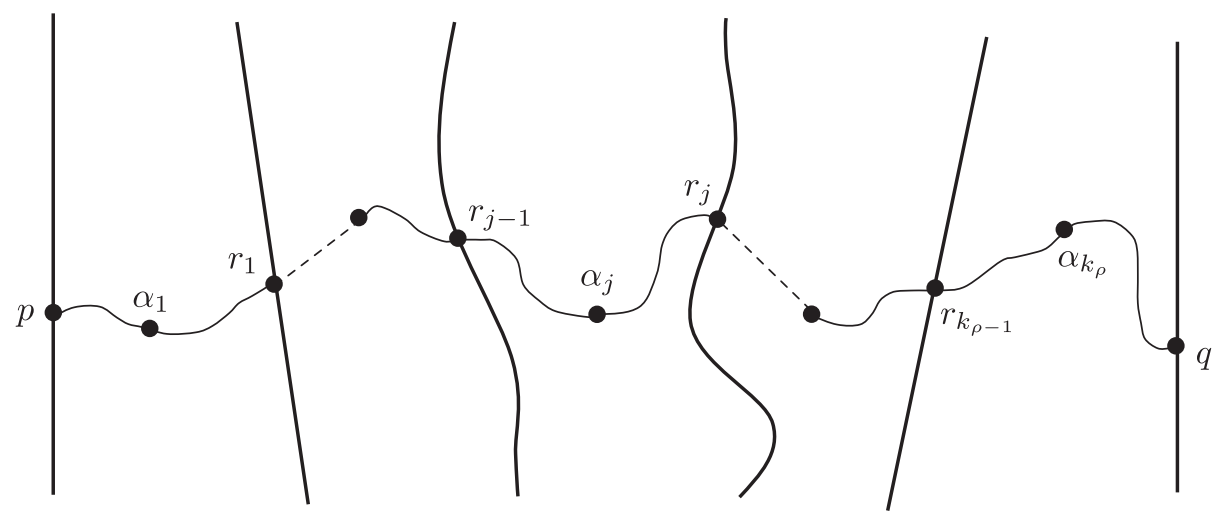

Fig. 1. Isolated periodic points located on a simple $\operatorname{arc} L_{p q}$. 


\section{Necessary definitions and facts}

\subsection{A-diffeomorphisms with an expanding attractor of codimension one}

Let $\mathbb{M}^{n}$ be a closed smooth orientable manifold of dimension $n>1, f: \mathbb{M}^{n} \rightarrow \mathbb{M}^{n}$ be a diffeomorphism and $N W(f)$ be its nonwandering set.

The diffeomorphism $f$ is said to satisfy an axiom $A$ (to be an A-diffeomorphism) if the set $N W(f)$ is hyperbolic and the periodic points are everywhere dense in $N W(f)$.

The following statement is called Smale's spectral decomposition theorem.

Proposition 1. Let $f: \mathbb{M}^{n} \rightarrow \mathbb{M}^{n}$ be an A-diffeomorphism. Then:

1) $N W(f)$ is uniquely represented as a finite union $N W(f)=\Lambda_{1} \cup \ldots \cup \Lambda_{m}$ of pairwise disjoint subsets of $\Lambda_{i}$, each of which is compact invariant and topologically transitive;

2) $\mathbb{M}^{n}=\bigcup_{i=1}^{m} W^{s}\left(\Lambda_{i}\right)=\bigcup_{i=1}^{m} W^{u}\left(\Lambda_{i}\right)$, where $W^{s}\left(\Lambda_{i}\right)=\left\{y \in \mathbb{M}^{n}: f^{k}(y) \rightarrow \Lambda_{i}, k \rightarrow+\infty\right\}$ and $W^{u}\left(\Lambda_{i}\right)=\left\{y \in \mathbb{M}^{n}: f^{-k}(y) \rightarrow \Lambda_{i}, k \rightarrow+\infty\right\}$.

The set $\Lambda_{i}$ is called a basic set. Using $\operatorname{dim} \Lambda_{i}$, denote its topological dimension.

Let $\Lambda$ be a basic set of an A-diffeomorphism $f$. The set $\Lambda$ is called an attractor (repeller) if it has a closed neighborhood $U_{\Lambda} \subset \mathbb{M}^{n}$ such that $f\left(U_{\Lambda}\right) \subset \operatorname{int} U_{\Lambda}, \bigcap_{k \in \mathbb{N}} f^{k}\left(U_{\Lambda}\right)=\Lambda$ $\left(f^{-1}\left(U_{\Lambda}\right) \subset \operatorname{int} U_{\Lambda}, \bigcap_{k \in \mathbb{N}} f^{-k}\left(U_{\Lambda}\right)=\Lambda\right)$. In this case, $\Lambda=\bigcup_{x \in \Lambda} W^{u}(x)\left(\Lambda=\bigcup_{x \in \Lambda} W^{s}(x)\right)$. If $\operatorname{dim} \Lambda=\operatorname{dim} W^{u}(x)\left(\operatorname{dim} \Lambda=\operatorname{dim} W^{s}(x)\right)$, then the attractor (repeller) $\Lambda$ is called expanding (contracting).

Due to [8, Theorem 3], any basic set $\Lambda$ of codimension one of an $A$-diffeomorphism $f: \mathbb{M}^{n} \rightarrow \mathbb{M}^{n}$ is either an attractor or a repeller. In this case $\Lambda$ is called orientable if for any point $x \in \Lambda$ and any fixed numbers $\alpha>0, \beta>0$ the index of intersection of local manifolds $W_{\alpha}^{s}(x) \cap W_{\beta}^{u}(x)$ is the same at all intersection points $(+1$ or -1$)$. Otherwise, the basic set $\Lambda$ is called nonorientable [2].

Everywhere below $\Lambda$ is an orientable expanding attractor of codimension one of an A-diffeomorphism $f: \mathbb{M}^{n} \rightarrow \mathbb{M}^{n}$.

Diffeomorphisms $f, g: M^{n} \rightarrow M^{n}$ are called topologically conjugate if there is a homeomorphism $h: M^{n} \rightarrow M^{n}$ such that $h \circ f=g \circ h$. If the last equality holds for a continuous map $h: M^{n} \rightarrow M^{n}$ (which is not a homeomorphism), then the diffeomorphisms $f, g$ are called semiconjugate.

A diffeomorphism $f$ is called structurally stable if there exists a neighborhood in the space of diffeomorphisms $M^{n} \rightarrow M^{n}$ such that any diffeomorphism from this neighborhood is topologically conjugate to the diffeomorphism $f$. By virtue of the results of R. Mañé [7] and C. Robinson [9], diffeomorphism $f$ is structurally stable if and only if it is an $A$-diffeomorphism and satisfies the strong transversality condition. The latter means that $\forall x, y \in N W(f)$ the stable manifold $W^{s}(x)$ of the point $x$ and the unstable manifold $W^{u}(y)$ of the point $y$ have only transversal intersections, that is, the sum of the tangent spaces to these invariant manifolds coincides with the tangent space to the ambient manifold at the intersection points.

For each point $x \in \Lambda$, the set $W^{s}(x) \backslash x$ consists of two connected components, and by virtue of [3], at least one of them has a nonempty intersection with the set $\Lambda$. Point $x \in \Lambda$ is called boundary if one of the connected components of the set $W^{s}(x) \backslash x$ does not intersect $\Lambda$. Let us denote this component by $W^{s \varnothing}(x)$.

RUSSIAN JOURNAL OF NONLINEAR DYNAMICS, 2020, 16(4), 595-606 
The set of boundary points of the basic set is finite. The union of the unstable manifolds $W^{u}\left(p_{1}\right), \ldots, W^{u}\left(p_{r_{b}}\right)$ boundary points $p_{1}, \ldots, p_{r_{b}}$ of the attractor $\Lambda$ whose components $W^{s \varnothing}\left(p_{1}\right), \ldots, W^{s \varnothing}\left(p_{r_{b}}\right)$ belong to the same path-connected component of the set $W^{s}(\Lambda) \backslash \Lambda$ is called a bunch $b$ of the attractor $\Lambda$. The number $r_{b}$ is called the degree of the bunch $b$. According to [4] and [6], if the diffeomorphism $f$ is given on the torus $\mathbb{T}^{n}$, then the attractor $\Lambda$ admits only 2-bunches and the pair of boundary points included in the bunch is called associated (see Fig. 2).
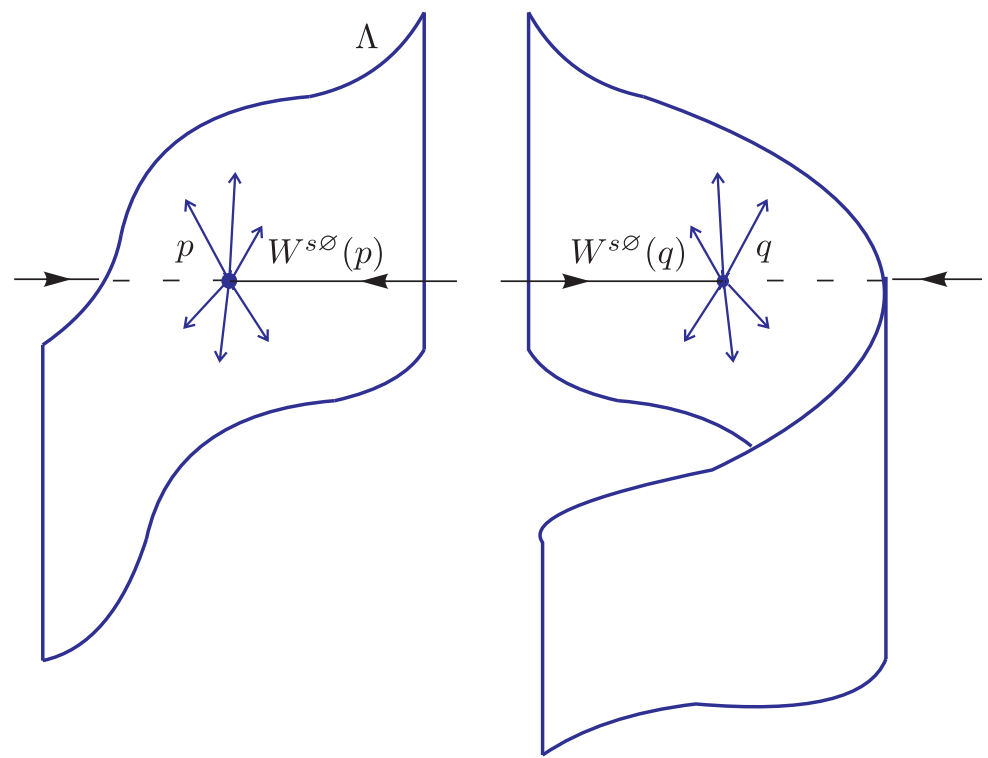

Fig. 2. 2-bunch of a two-dimensional expanding attractor $\Lambda$ on a 3-manifold.

In [6] it is proved that, if $f: \mathbb{M}^{n} \rightarrow \mathbb{M}^{n}(n \geqslant 3)$ is a structurally stable diffeomorphism whose nonwandering set $N W(f)$ contains an expanding orientable attractor $\Lambda$ of codimension one, then:

- the manifold $\mathbb{M}^{n}$ is homotopically equivalent to the torus $\mathbb{T}^{n}$, and for $n \neq 4, \mathbb{M}^{n}$ is homeomorphic to $\mathbb{T}^{n}$ ([6, Theorem 5.1]);

- the set $N W(f) \backslash \Lambda$ consists of a finite number of isolated sources and saddles, and the union of the stable manifolds of isolated points closure and components $W^{s \varnothing(p)}, W^{s \varnothing}(q)$ splits into a finite number of simple arcs, each of which contains a finite nonzero number of sources, a finite (possibly zero) number of saddle points, and exactly two associated boundary points of the attractor $\Lambda$ ([6, Corollary 5.2], see Fig. 3).

Note that the attractor orientability requirement can be omitted in the case $n=3$ by [10].

For the case $n=2$, any one-dimensional basic set of an $A$-diffeomorphism is either an expanding attractor or a contracting repeller. However, the statements formulated above are not true in dimension two. Namely, the ambient surface $\mathbb{M}^{2}$ of an $A$-diffeomorphism $f$ whose nonwandering set $N W(f)$ contains an orientable attractor $\Lambda$ is not necessarily a torus, such diffeomorphisms admit any orientable surfaces other than 2-sphere. In the case where $\mathbb{M}^{2}=\mathbb{T}^{2}$, according to [4], the set $N W(f) \backslash \Lambda$ consists of a finite number of isolated periodic points, which can be located on simple arcs (see Fig. 1) or have other disposition (see Fig. 4). 


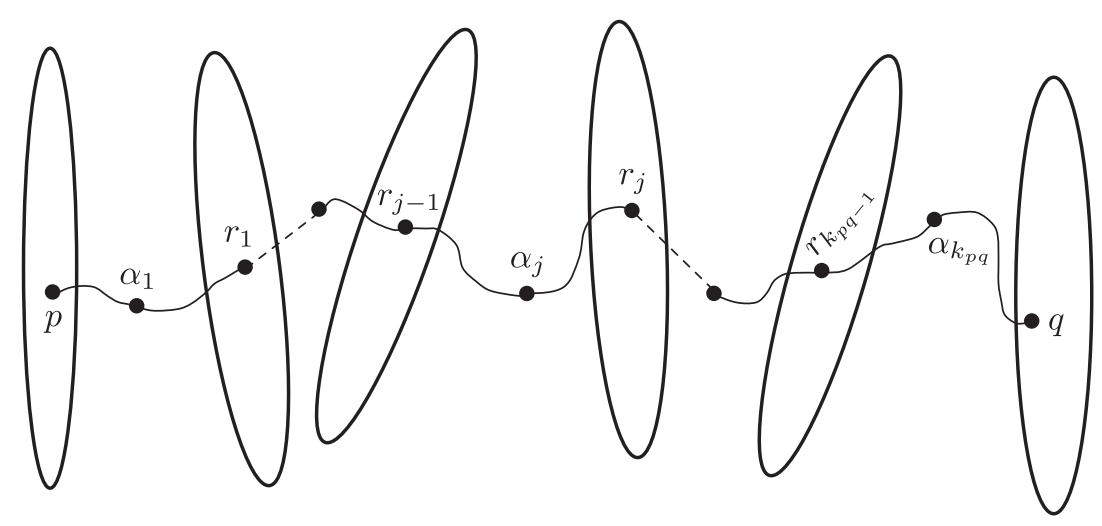

Fig. 3. Associated arc $L_{p q}$.

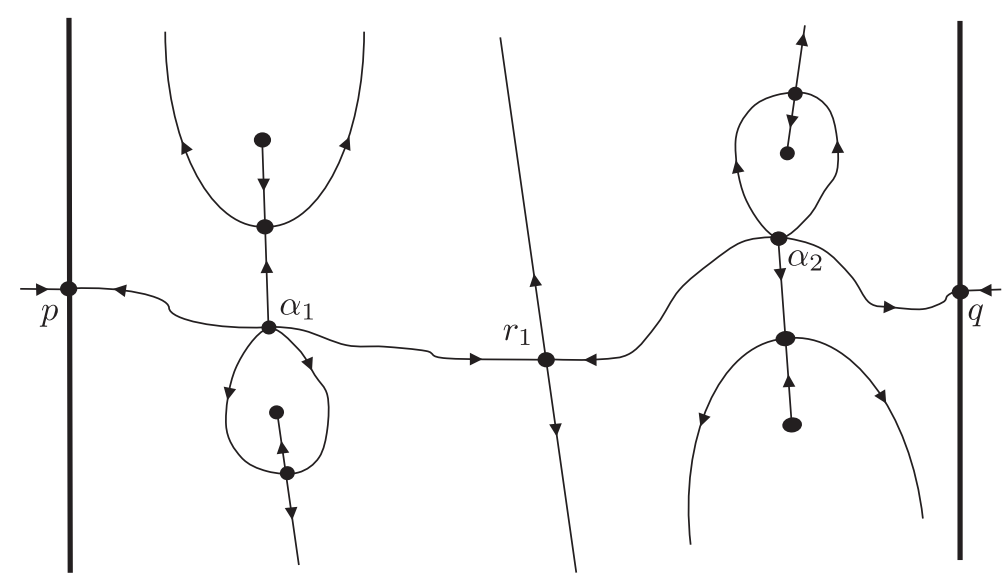

Fig. 4. Isolated periodic points outside the associated arc.

\subsection{Orientable expanding attractors on a 2-torus}

It is known that any diffeomorphism $f$ on a two-dimensional torus induces an automorphism of the fundamental group $f_{*}: \pi_{1}\left(\mathbb{T}^{2}\right) \rightarrow \pi_{1}\left(\mathbb{T}^{2}\right)$, where the group $\pi_{1}\left(\mathbb{T}^{2}\right)$ is isomorphic to the Abelian group $\mathbb{Z}^{2}$. Then the automorphism $f_{*}$ is uniquely determined by the matrix $A_{f}$ belonging to the set $G L(2, \mathbb{Z})$ of unimodular integer matrices. An automorphism $f_{*}$ is called hyperbolic if the matrix $A_{f}$ is hyperbolic, i.e., it has no eigenvalues with modulus one.

An algebraic automorphism $\widehat{A}: \mathbb{T}^{2} \rightarrow \mathbb{T}^{2}$ of the torus $\mathbb{T}^{2}=\mathbb{R}^{2} / \mathbb{Z}^{2}$ is a diffeomorphism defined by the matrix $A=\left(\begin{array}{ll}\alpha & \beta \\ \gamma & \delta\end{array}\right) \in G L(2, \mathbb{Z})$, i.e.,

$$
\widehat{A}(x, y)=(\alpha x+\beta y, \gamma x+\delta y) \quad(\bmod 1) .
$$

If the matrix $A$ is hyperbolic, then $\widehat{A}$ is an Anosov diffeomorphism, that is, the hole ambient manifold $M^{2}$ is its hyperbolic set.

Proposition 2 ([1]). Let $f: \mathbb{T}^{2} \rightarrow \mathbb{T}^{2}$ be a diffeomorphism and let the induced isomorphism $f_{*}: \pi_{1}\left(\mathbb{T}^{2}\right) \rightarrow \pi_{1}\left(\mathbb{T}^{2}\right)$ be hyperbolic. Then among the homotopic to the identity continuous maps of the torus $\mathbb{T}^{2}$ there is a unique map $h_{f}$ that semiconjugates the diffeomorphism $f$ with the algebraic automorphism $\widehat{A}_{f}$ (see the diagram in Fig. 5). In this case, if $f$ is an Anosov diffeomorphism, then $h_{f}$ is a homeomorphism. 


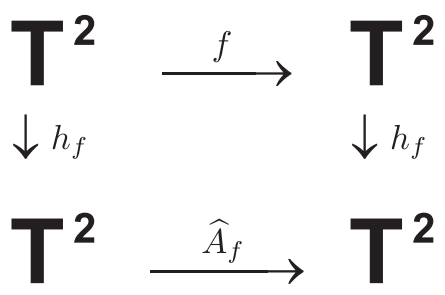

Fig. 5. Semi-conjugation.

Proposition $3([4,5])$. If an A-diffeomorphism $f: \mathbb{T}^{2} \rightarrow \mathbb{T}^{2}$ has an orientable basic set $\Lambda$, then the induced automorphism $f_{*}: \pi_{1}\left(\mathbb{T}^{2}\right) \rightarrow \pi_{1}\left(\mathbb{T}^{2}\right)$ is hyperbolic.

Proposition $4([4,5])$. If $\Lambda$ is an orientable basic set of an $A$-diffeomorphism $f: \mathbb{T}^{2} \rightarrow \mathbb{T}^{2}$, then $f$ has no orientable basic sets other than $\Lambda$.

Let $\Lambda$ be a one-dimensional orientable attractor of the diffeomorphism $f: \mathbb{T}^{2} \rightarrow \mathbb{T}^{2}$ and $h_{f}: \mathbb{T}^{2} \rightarrow \mathbb{T}^{2}$ be the semiconjugating map to the algebraic automorphism $\hat{A}_{f}$. Let $B_{f}=$ $=\left\{x \in \mathbb{T}^{2}: h_{f}^{-1}(x)\right.$ consist of more than one point $\}$.

Proposition $5([4,5])$. The image $h_{f}(\Lambda)$ of the set $\Lambda$ is the whole torus $\mathbb{T}^{2}$. The set $B_{f}$ is the union of a finitely many periodic points $P_{f}=\left\{\varrho_{1}, \varrho_{2}, \ldots, \varrho_{k}\right\}$ of the algebraic automorphism $\widehat{A}_{f}$ and their unstable manifolds. The set $h_{f}^{-1}\left(\varrho_{i}\right) \cap \Lambda, i \in\{1,2, \ldots, k\}$, consists of two boundary points $p_{i}, q_{i}$ of the set $\Lambda$.

Proposition $6([4,5])$. Let $f, f^{\prime}: \mathbb{T}^{2} \rightarrow \mathbb{T}^{2}$ be A-diffeomorphisms and $\Lambda, \Lambda^{\prime}$ be their orientable attractors, respectively. Then there is a homeomorphism $\varphi: \mathbb{T}^{2} \rightarrow \mathbb{T}^{2}$ for which $\varphi(\Lambda)=\Lambda^{\prime},\left.f^{\prime}\right|_{\Lambda^{\prime}}=\left.\varphi f \varphi^{-1}\right|_{\Lambda^{\prime}}$ if and only if there is a matrix $H \in G L(2, \mathbb{Z})$ such that $H A_{f}=$ $=A_{f^{\prime}} H$ and $\widehat{H}\left(P_{f}\right)=P_{f^{\prime}}$.

\section{Proof of conjugation criteria (proof of Theorem 1)}

Consider a structurally stable diffeomorphism $f: \mathbb{T}^{2} \rightarrow \mathbb{T}^{2}$ from class $G$. We describe the properties of the diffeomorphism $f$, which will be used substantially in the proof of the theorem.

The nonwandering set of the diffeomorphism $f \in G$ contains a unique nontrivial basic set $\Lambda$ which is a one-dimensional orientable expanding attractor. For different points $a, b \in W^{s}(x)$, $x \in \Lambda$ we denote by $[a, b]^{s}$ a compact segment of the manifold $W^{s}(x)$ bounded by the points $a, b$. Let $(a, b)^{s}=[a, b]^{s} \backslash(a \cup b)$.

The set $\Gamma_{\Lambda}$ of all boundary points of the set $\Lambda$ is not empty and consists of a finite number of periodic points that are split into associated pairs $\left(p_{i}, q_{i}\right), i=1, \ldots, k_{f}$ of points of the same period so that the bunch $B_{p_{i} q_{i}}=W^{u}\left(p_{i}\right) \cup W^{u}\left(q_{i}\right)$ is accessible from within the boundary ${ }^{1}$ of some connected component $G_{p_{i} q_{i}}$ of the set $\mathbb{T}^{2} \backslash \Lambda$. Denote by $B_{\Lambda}$ the set of all bunches of the basic set $\Lambda$.

Let $T(f)=N W(f) \backslash \Lambda$ and $T_{p_{i} q_{i}}=T(f) \cap G_{p_{i} q_{i}}$. Closures of the stable manifolds of isolated periodic points from the set $T_{p_{i} q_{i}}$ belong to simple arcs $L_{p_{i} q_{i}}$ bounded by pairs of boundary

\footnotetext{
${ }^{1}$ Let $V \subset M$ be an open set with boundary $\partial V(\partial V=c l(V) \backslash \operatorname{int}(V))$. A subset $\delta V \subset \partial V$ is called accessible from within the domain $V$ if for any point $x \in \delta V$ there is an open arc that lies completely in $V$ and such that $x$ is one of its endpoints.
} 
points $p_{i}, q_{i}$. For any point $x \in W^{u}\left(p_{i}\right) \backslash p_{i}$, there is a unique point $y \in\left(W^{u}\left(q_{i}\right) \cap W^{s}(x)\right)$ such that the $\operatorname{arc}(x, y)^{s}$ does not intersect $\Lambda$. Define a map

$$
\xi_{p_{i} q_{i}}: B_{p_{i} q_{i}} \backslash\left\{p_{i}, q_{i}\right\} \rightarrow B_{p_{i} q_{i}} \backslash\left\{p_{i}, q_{i}\right\},
$$

assuming $\quad \xi_{p_{i} q_{i}}(x)=y \quad$ and $\quad \xi_{p_{i} q_{i}}(y)=x . \quad$ Then $\quad \xi_{p_{i} q_{i}}\left(W^{u}\left(p_{i}\right) \backslash p_{i}\right)=W^{u}\left(q_{i}\right) \backslash q_{i} \quad$ and $\xi_{p_{i} q_{i}}\left(W^{u}\left(q_{i}\right) \backslash q_{i}\right)=W^{u}\left(p_{i}\right) \backslash p_{i}$, i.e., the map $\xi_{p_{i} q_{i}}$ maps punctured unstable 2-bunch manifolds to each other and is an involution $\left(\xi_{p_{i} q_{i}}^{2}(x)=i d\right)$. By virtue of the theorem of continuous dependence of invariant manifolds on compact sets, the map $\xi_{p_{i} q_{i}}$ is a homeomorphism.

Denote by $m_{p_{i}}$ the period of the point $p_{i}$ and by $m_{i}$ the period of the component $G_{p_{i} q_{i}}$. Then the restriction $\left.f^{m_{p_{i}}}\right|_{W^{u}\left(p_{i}\right)}$ has exactly one hyperbolic repelling fixed point $p_{i}$, so there is a smooth closed segment $D_{p_{i}} \subset W^{u}\left(p_{i}\right)$ such that $p_{i} \in D_{p_{i}} \subset \operatorname{int}\left(f^{m_{p_{i}}}\left(D_{p_{i}}\right)\right)$. Then the set $C_{p_{i} q_{i}}=\bigcup_{x \in \partial D_{p_{i}}}\left[x, \xi_{p_{i} q_{i}}(x)\right]^{s}$ consists of two segments and the points $\xi_{p_{i} q_{i}}\left(\partial D_{p_{i}}\right)$ bounded in $W^{u}\left(q_{i}\right)$ segment $D_{q_{i}}$ such that $q_{i} \in D_{q_{i}} \subset \operatorname{int}\left(f^{m_{i}}\left(D_{q_{i}}\right)\right)$. The set $S_{p_{i} q_{i}}=D_{p_{i}} \cup C_{p_{i} q_{i}} \cup D_{q_{i}}$ is homeomorphic to a circle. We will call $S_{p_{i} q_{i}}$ the characteristic circle corresponding to the bunch $B_{p_{i} q_{i}}$. Each set $S_{p_{i} q_{i}}$ bounds a two-dimensional disk $Q_{p_{i} q_{i}}$ such that the set $L_{p_{i} q_{i}}$ containing periodic points from the set $T_{p_{i} q_{i}}$ is a subset of $Q_{p_{i} q_{i}}$.

The induced isomorphism $f_{*}: \pi_{1}\left(\mathbb{T}^{2}\right) \rightarrow \pi_{1}\left(\mathbb{T}^{2}\right)$ is uniquely defined by the hyperbolic matrix $A_{f} \in G L(2, \mathbb{Z})$, and among the homotopic to identity continuous maps of the torus $\mathbb{T}^{2}$ there is a unique map $h_{f}$ that semiconjugates the diffeomorphism $f$ with the diffeomorphism $\widehat{A}_{f}$. The map $h_{f}$ sends the set $\Lambda$ to the whole torus $\mathbb{T}^{2}$, and the set $B_{f}=\left\{x \in \mathbb{T}^{2}: h_{f}^{-1}(x)\right.$ consisting of more than one point $\}$ consists of a finite number of periodic points $P_{f}=\left\{\varrho_{1}, \varrho_{2}, \ldots, \varrho_{k}\right\}$ of the diffeomorphism $\widehat{A}_{f}$ and their unstable manifolds. In this case, $h_{f}^{-1}\left(\varrho_{i}\right) \cap \Lambda, i \in\{1,2, \ldots, k\}$, consists of a pair of boundary associated points $p_{i}, q_{i}$ of the basic set $\Lambda$ and $h_{f}\left(L_{p_{i} q_{i}}\right)=\varrho_{i}$. Denote by $n_{\varrho_{i}}$ the number of sources on the $\operatorname{arc} L_{p_{i} q_{i}}$.

In addition to the diffeomorphism $f$, we consider the diffeomorphism $f^{\prime} \in G$, whose basic set contains a one-dimensional orientable expanding attractor $\Lambda^{\prime}$; we provide strokes for all other objects considered in connection with the mapping $f^{\prime}$.

We prove that the diffeomorphisms $f, f^{\prime} \in G$ are topologically conjugate if and only if there exists a matrix $H \in G L(2, \mathbb{Z})$ such that $H A_{f}=A_{f^{\prime}} H, \widehat{H}\left(P_{f}\right)=P_{f^{\prime}}$ and $n_{\varrho_{i}}=n_{\widehat{H}\left(\varrho_{i}\right)}$, $i=1, \ldots, k$.

\subsection{Necessity}

If the diffeomorphisms $f, f^{\prime} \in G$ are topologically conjugate, then there is a homeomorphism $h: \mathbb{T}^{2} \rightarrow \mathbb{T}^{2}$ such that $h f=f^{\prime} h$. The induced isomorphism of $h_{*}: \pi_{1}\left(\mathbb{T}^{2}\right) \rightarrow \pi_{1}\left(\mathbb{T}^{2}\right)$ is uniquely defined by the hyperbolic matrix $H \in G L(2, \mathbb{Z})$, which by conjugation satisfies the condition $H A_{f}=A_{f^{\prime}} H$ and consequently the condition $\widehat{H} \widehat{A}_{f}=\widehat{A}_{f^{\prime}} \widehat{H}$.

Since $h$ is a conjugating homeomorphism, it converts the boundary points $p_{i}$ of the diffeomorphism $f$ to the boundary points of the diffeomorphism $f^{\prime}$. Let us say $p_{i}^{\prime}=h\left(p_{i}\right)$ and $q_{i}^{\prime}=h\left(q_{i}\right)$

It follows from the semiconjugations that $h_{f} f=\widehat{A}_{f} h_{f}$ and $h_{f^{\prime}} f^{\prime}=\widehat{A}_{f^{\prime}} h_{f^{\prime}}$. Since $h_{f}\left(p_{i}\right)=\varrho_{i}$, it follows that $h_{f}\left(f\left(p_{i}\right)\right)=\widehat{A}_{f}\left(\varrho_{i}\right)$ and hence $\widehat{A}_{f}\left(P_{f}\right)=P_{f}$. Similarly, $h_{f^{\prime}}\left(p_{i}^{\prime}\right)=\varrho_{i}^{\prime}, h_{f^{\prime}}\left(f^{\prime}\left(p_{i}^{\prime}\right)\right)=$ $=\widehat{A}_{f^{\prime}}\left(\varrho_{i}^{\prime}\right)$ and hence $\widehat{A}_{f^{\prime}}\left(P_{f^{\prime}}\right)=P_{f^{\prime}}$. So $\widehat{H}\left(P_{f}\right)=P_{f^{\prime}}$ and $\widehat{H}\left(\varrho_{i}\right)=\varrho_{i}^{\prime}$.

Since $h$ is a conjugating homeomorphism, the number $n_{\varrho_{i}}$ of sources on the $\operatorname{arc} L_{p_{i} q_{i}}$ coincides with the number $n_{\varrho_{i}^{\prime}}$ of sources on the $\operatorname{arc} L_{p_{i} q_{i}^{\prime}}$, where $n_{\varrho_{i}}=n_{\widehat{H}\left(\varrho_{i}\right)}, i=1, \ldots, k$. 


\subsection{Sufficiency}

Let $f, f^{\prime} \in G$ and suppose there exists a matrix $H \in G L(2, \mathbb{Z})$ such that $H A_{f}=A_{f^{\prime}} H$, $\widehat{H}\left(P_{f}\right)=P_{f^{\prime}}$ and $n_{\varrho_{i}}=n_{\widehat{H}\left(\varrho_{i}\right)}, i=1, \ldots, k$. Let us construct a homeomorphism $\varphi: \mathbb{T}^{2} \rightarrow \mathbb{T}^{2}$ conjugating the diffeomorphisms $f$ and $f^{\prime}$. We will construct a conjugation step by step.

\subsubsection{Conjugation on nontrivial basic sets}

Semiconjugation $h_{f}$ maps the attractor $\Lambda$ to the hole torus $\mathbb{T}^{2}$, with $h_{f}\left(L_{p_{i} q_{i}}\right)=\varrho_{i}$. A similar action is performed by semiconjugating $h_{f^{\prime}}$ with the attractor $\Lambda^{\prime}$. Moreover, the maps $q_{f}=\left.h_{f}\right|_{\Lambda \backslash B_{\Lambda}}: \Lambda \backslash B_{\Lambda} \rightarrow \mathbb{T}^{2} \backslash W^{u}\left(P_{f}\right), q_{f^{\prime}}=\left.h_{f^{\prime}}\right|_{\Lambda^{\prime} \backslash B_{\Lambda^{\prime}}}: \Lambda^{\prime} \backslash B_{\Lambda^{\prime}} \rightarrow \mathbb{T}^{2} \backslash W^{u}\left(P_{f^{\prime}}\right)$ are homeomorphisms. By condition $\widehat{H}\left(P_{f}\right)=P_{f^{\prime}}$, this means that the map

$$
\varphi=q_{f^{\prime}}^{-1} \widehat{H} q_{f}: \Lambda \backslash B_{\Lambda} \rightarrow \Lambda^{\prime} \backslash B_{\Lambda^{\prime}}
$$

is a homeomorphism conjugating $f$ and $f^{\prime}$ by [4] (see also [5, pp. 224-225]).

We show how to continue $\varphi$ to the set $B_{\Lambda}$. For the boundary point $p \in \Gamma_{\Lambda}\left(p^{\prime} \in \Gamma_{\Lambda^{\prime}}\right)$ denote by $W^{s \infty}(p)\left(W^{s \infty}\left(p^{\prime}\right)\right)$ the stable separatrix of the point $p\left(p^{\prime}\right)$ that has a nonempty intersection with $\Lambda\left(\Lambda^{\prime}\right)$. From the fact that $\varphi$ is a conjugating homeomorphism, it follows that $\varphi\left(W^{s \infty}\left(\Gamma_{\Lambda}\right)\right)=W^{s \infty}\left(\Gamma_{\Lambda^{\prime}}\right)$. Thus, the homeomorphism $\varphi$ is uniquely extended to the set $\Gamma_{\Lambda}$. Since $h_{f}\left(W^{s, \infty}\left(p_{i}\right) \cup W^{s \infty}\left(q_{i}\right)\right)=W^{s}\left(\varrho_{i}\right) \backslash \varrho_{i}$ for paired points $p_{i}, q_{i}$ and $\widehat{H}\left(\varrho_{i}\right)=\varrho_{i}^{\prime}$, it follows that $p_{i}^{\prime}=\varphi\left(p_{i}\right), q_{i}^{\prime}=\varphi\left(q_{i}\right)$ are paired points such that $\varrho_{i}^{\prime}=h_{f^{\prime}}\left(L_{p_{i}^{\prime} q_{i}^{\prime}}\right)$.

Since for any point $x \in W^{u}\left(\Gamma_{\Lambda}\right)$ there is such a sequence of points $x_{n} \in W^{s \infty}(x) \cap\left(\Lambda \backslash B_{\Lambda}\right)$ for which $x$ is a limit point, then the homeomorphism $\varphi$ can be continued to the set $W^{u}\left(\Gamma_{\Lambda}\right)$ by the formula $x^{\prime}=\varphi(x)$, where $x^{\prime}$ is the limit point of the sequence of points $\varphi\left(x_{n}\right)$.

An exact proof of the fact that the map $\varphi$ is a homeomorphism conjugating the basic sets $\Lambda$ and $\Lambda^{\prime}$ of $f$ and $f^{\prime}$, respectively, at all points of the above-mentioned sets, is given in [4] (see also [5, pp. 224-225]). Thus, to prove Theorem 1, it is sufficient to continue the map $\varphi$ to the set $\mathbb{T}^{2} \backslash \Lambda$.

\subsubsection{Conjugation on trivial basic sets}

For each associated pair of boundary points $\left(p_{i}, q_{i}\right)$ there is a natural number $n_{\varrho_{i}}$ such that the set of isolated periodic points $T_{p_{i} q_{i}}$ consists of exactly $n_{\varrho_{i}}$ periodic sources $\alpha_{1}^{i}, \ldots, \alpha_{n_{\varrho_{i}}}^{i}$ and $n_{\varrho_{i}}-1$ periodic saddle points $r_{1}^{i}, \ldots, r_{n_{\varrho_{i}}-1}^{i}$, alternating with sources on a simple arc $L_{p_{i} q_{i}}=W^{s \varnothing}\left(p_{i}\right) \cup \bigcup_{j=1}^{n_{\varrho_{i}}-1} W^{s}\left(r_{j}^{i}\right) \cup \bigcup_{j=1}^{n_{\varrho_{i}}} \alpha_{j}^{i} \cup W^{s \varnothing}\left(q_{i}\right)$ from the point $p_{i}$ to the point $q_{i}$ (see Fig. 1). Similarly, by primes we denote the periodic points of the arc $L_{p_{i}^{\prime} q_{i}^{\prime}}$. Since $n_{\varrho_{i}}=n_{\varrho_{i}^{\prime}}$, the equality $\varphi_{i}\left(\alpha_{j}^{i}\right)=\alpha_{j}^{i}, \varphi_{i}\left(r_{j}^{i}\right)=r_{j}^{\prime i}$ defines a homeomorphism $\varphi_{i}: T_{p_{i} q_{i}} \rightarrow T_{p_{i}^{\prime} q_{i}^{\prime}}$. Denote by $\varphi_{T}: T(f) \rightarrow T\left(f^{\prime}\right)$ a homeomorphism composed by $\varphi_{1}, \ldots, \varphi_{k}$.

We show that $\varphi_{T}$ conjugates the diffeomorphisms $\left.f\right|_{T(f)}$ and $\left.f^{\prime}\right|_{T\left(f^{\prime}\right)}$.

Since the diffeomorphism $\widehat{H}$ conjugates the diffeomorphisms $\widehat{A}_{f}, \widehat{A}_{f^{\prime}}$ and $\widehat{H}\left(\varrho_{i}\right)=\varrho_{i}^{\prime}$, the periods of the points $\varrho_{i}$ and $\varrho_{i}^{\prime}$ are $m_{i}$, where $m_{i}$ is the period of the connectivity component $G_{p_{i}} q_{i}$ $\left(G_{p_{i}^{\prime} q_{i}^{\prime}}\right)$ of the diffeomorphism $f\left(f^{\prime}\right)$. Since $h_{f}\left(L_{p_{i} q_{i}}\right)=\varrho_{i}$ and $h_{f^{\prime}}\left(L_{p_{i}^{\prime} q_{i}^{\prime}}\right)=\varrho_{i}^{\prime}$, the arcs $L_{p_{i} q_{i}}$ and $L_{p_{i}^{\prime} q_{i}^{\prime}}$ also have a period $m_{i}$. In this case, the diffeomorphism $\left.f^{m_{i}}\right|_{L_{p_{i} q_{i}}}$ preserves (changes) the orientation if the eigenvalue $\lambda$ of the matrix $A_{f}$ is positive (negative). Similarly, for the diffeomorphism $\left.f^{\prime m_{i}}\right|_{L_{p_{i}^{\prime} q_{i}^{\prime}}}$ Thus, if $\lambda>0$, then all periodic points of the diffeomorphism $\left.f^{m_{i}}\right|_{L_{p_{i} q_{i}}}$ 
are fixed; otherwise, there is exactly one fixed point, and all the others have a period of two. By construction, $\varphi_{T}\left(L_{p_{i} q_{i}} \cap T(f)\right)=L_{\varphi\left(p_{i}\right) \varphi\left(q_{i}\right)} \cap T\left(f^{\prime}\right)$ and the homeomorphism $\varphi$ conjugates $\left.f\right|_{\Gamma_{\Lambda}}$ and $\left.f^{\prime}\right|_{\Gamma_{\Lambda^{\prime}}}$, hence the homeomorphism $\varphi_{T}$ conjugates the diffeomorphisms $\left.f\right|_{T(f)}$ and $\left.f^{\prime}\right|_{T\left(f^{\prime}\right)}$.

The homeomorphism $\varphi_{T}$ naturally continues to unstable manifolds of isolated saddle points. Exactly, let $z \in W^{u}\left(r_{j}^{i}\right) \backslash r_{j}^{i}$. Then there is a unique point $x_{z} \in W^{u}\left(p_{i}\right) \backslash p_{i}$ such that $z=\left[x_{z}, \xi_{p_{i} q_{i}}\left(x_{z}\right)\right]^{s} \cap W^{u}\left(r_{j}^{i}\right)$. The same is true for the diffeomorphism $f^{\prime}$. Let

$$
\varphi_{T}(z)=z^{\prime}=\left[x_{z}^{\prime}, \xi_{p_{i}^{\prime} q_{i}^{\prime}}\left(x_{z}^{\prime}\right)\right]^{s} \cap W^{u}\left(r_{j}^{i}\right)
$$

and $\varphi_{T} f^{m_{i}}(z)=f^{\prime m_{i}} \varphi_{T}(z)$ if $\lambda>0 ; \varphi_{T} f^{2 m_{i}}(z)=f^{\prime 2 m_{i}} \varphi_{T}(z)$ if $\lambda<0$.

\subsubsection{Conjugation in source basins}

Consider the source $\alpha_{j}^{i}$ and put for convenience $p_{i}=r_{0}^{i}, q_{i}=r_{n_{\varrho_{i}}}^{i}$. On the stable separatrices $\ell_{j-1}^{i}, \ell_{j}^{i}$ of the saddles $r_{j-1}^{i}, r_{j}^{i}$ lying in the basin of $\alpha_{j}^{i}$, we select points $y_{j-1}^{i}, y_{j}^{i}$ and $\operatorname{arcs} v_{j-1}^{i}, v_{j}^{i}$ transversally intersecting the separatrices at these points. By $\lambda$-lemma, we can draw these arcs so that each segment $\left[x, \xi_{p_{i} q_{i}}(x)\right]^{s}, x \in\left(D_{p_{i}} \backslash p_{i}\right)$ transversally intersects each of the $\operatorname{arcs} v_{j-1}^{i}, v_{j}^{i}$ at a unique point. Denote by $Q_{j}^{i}$ the closure of the connected component of the set $Q_{p_{i} q_{i}} \backslash\left(v_{j-1}^{i} \cup v_{j}^{i}\right)$ containing $\alpha_{j}^{i}$. Let $\phi=f^{m_{i}}$.

Let $x_{1}, x_{2}$ denote the boundary points of the segment $D_{p_{i}}$. Let $\xi_{p_{i} q_{i}}\left(x_{1}\right)=x_{4} \in W^{u}\left(q_{i}\right)$ and $\xi_{p_{i} q_{i}}\left(x_{2}\right)=x_{3} \in W^{u}\left(q_{i}\right)$. So $\left(x_{2}, x_{3}\right)^{s} \cap \Lambda=\varnothing,\left(x_{1}, x_{4}\right)^{s} \cap \Lambda=\varnothing$, and both arcs intersect the manifold $W^{u}\left(\alpha_{j}^{i}\right)$. Let $A=\left(x_{1}, x_{4}\right)^{s} \cap v_{j-1}^{i}, B=\left(x_{2}, x_{3}\right)^{s} \cap v_{j-1}^{i}, C=\left(x_{2}, x_{3}\right)^{s} \cap v_{j}^{i}$, $D=\left(x_{1}, x_{4}\right) \cap v_{j}^{i}$. Then the closed curve $L_{j}^{i}=A B C D$ is the boundary of the domain $Q_{j}^{i}$. Let $E=\phi(A), F=\phi(B), G=\phi(C), H=\phi(D)$ (Figure 6 shows a case in which $\phi\left(p_{i}\right)=p_{i}$, in the case of $\phi\left(p_{i}\right)=q_{i}$ the reasoning is repeated verbatim). Then the closed curve $\phi\left(L_{j}^{i}\right)=E F G H$ is the boundary of the domain $\phi\left(Q_{j}^{i}\right)$. Let $K_{j}^{i}=c l\left(\phi\left(Q_{j}^{i}\right) \backslash Q_{j}^{i}\right)$. It is obvious that the annulus $K_{j}^{i}$ is a fundamental domain of the diffeomorphism $\phi$ restricted to $W^{u}\left(\alpha_{j}^{i}\right) \backslash \alpha_{j}^{i}$.

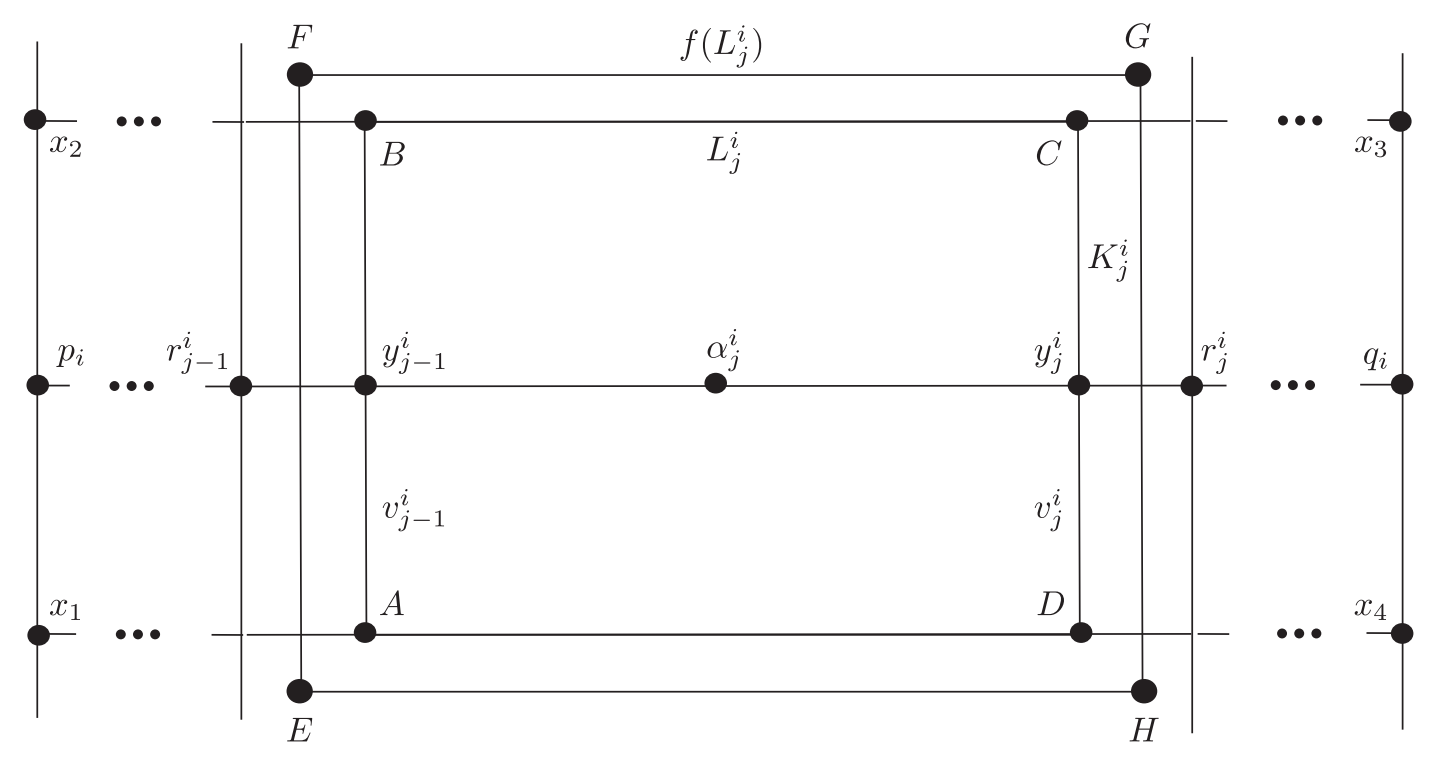

Fig. 6. Fundamental domain of the diffeomorphism $f$. 
Let us add a prime to similar objects for the diffeomorphism $f^{\prime}$, assuming $p_{i}^{\prime}=\varphi\left(p_{i}\right)$, $q_{i}^{\prime}=\varphi\left(q_{i}\right), x_{i}^{\prime}=\varphi\left(x_{i}\right)$. By construction, for any point $v \in\left(A B \backslash W^{s}\left(r_{j-1}^{i}\right)\right)$ there is a unique point $x_{v} \in\left(W^{u}\left(p_{i}\right) \backslash p_{i}\right)$ such that $v=\left(x_{v}, \xi_{p_{i} q_{i}}\left(x_{v}\right)\right)^{s} \cap A B$. Define the homeomorphism $\varphi_{A B}: A B \rightarrow A^{\prime} b^{\prime}$ by the formula $\varphi_{A B}(v)=v^{\prime} \in A^{\prime} b^{\prime}$, where $v^{\prime}=\left(x_{v}^{\prime}, \xi_{p_{i}^{\prime} q_{i}^{\prime}}\left(x_{v}^{\prime}\right)\right)^{s} \cap a^{\prime} b^{\prime}$ and $\left.\varphi_{A B}\left(A B \cap W^{s}\left(r_{j-1}^{i}\right)\right)=A^{\prime} B^{\prime} \cap W^{s}\left(r_{j-1}^{\prime i}\right)\right)$ (see Fig. 7). Similarly, we construct the homeomorphism $\varphi_{C D}: C D \rightarrow C^{\prime} D^{\prime}$. Finally, we compose a homeomorphism $\varphi_{L_{j}^{i}}: L_{j}^{i} \rightarrow L_{j}^{\prime i}$. Let $\varphi_{\phi\left(L_{j}^{i}\right)}=$ $=\phi \varphi_{L_{j}^{i}} \phi^{-1}: \phi\left(L_{j}^{i}\right) \rightarrow \phi^{\prime}\left(L_{j}^{\prime i}\right)$. In the same way, we construct homeomorphisms $\varphi_{A E}: A E \rightarrow A^{\prime} E^{\prime}$, $\varphi_{B F}: B F \rightarrow B^{\prime} f^{\prime}, \varphi_{C G}: C G \rightarrow C^{\prime} G^{\prime}$ and $\varphi_{D H}: D H \rightarrow D^{\prime} H^{\prime}$, where all segments are chosen transversally to the foliation $W^{s}(\Lambda)$. Denote by $\varphi_{\partial}$ the union of the constructed homeomorphisms. Let $x^{\prime}=\varphi_{\partial}(x)$.

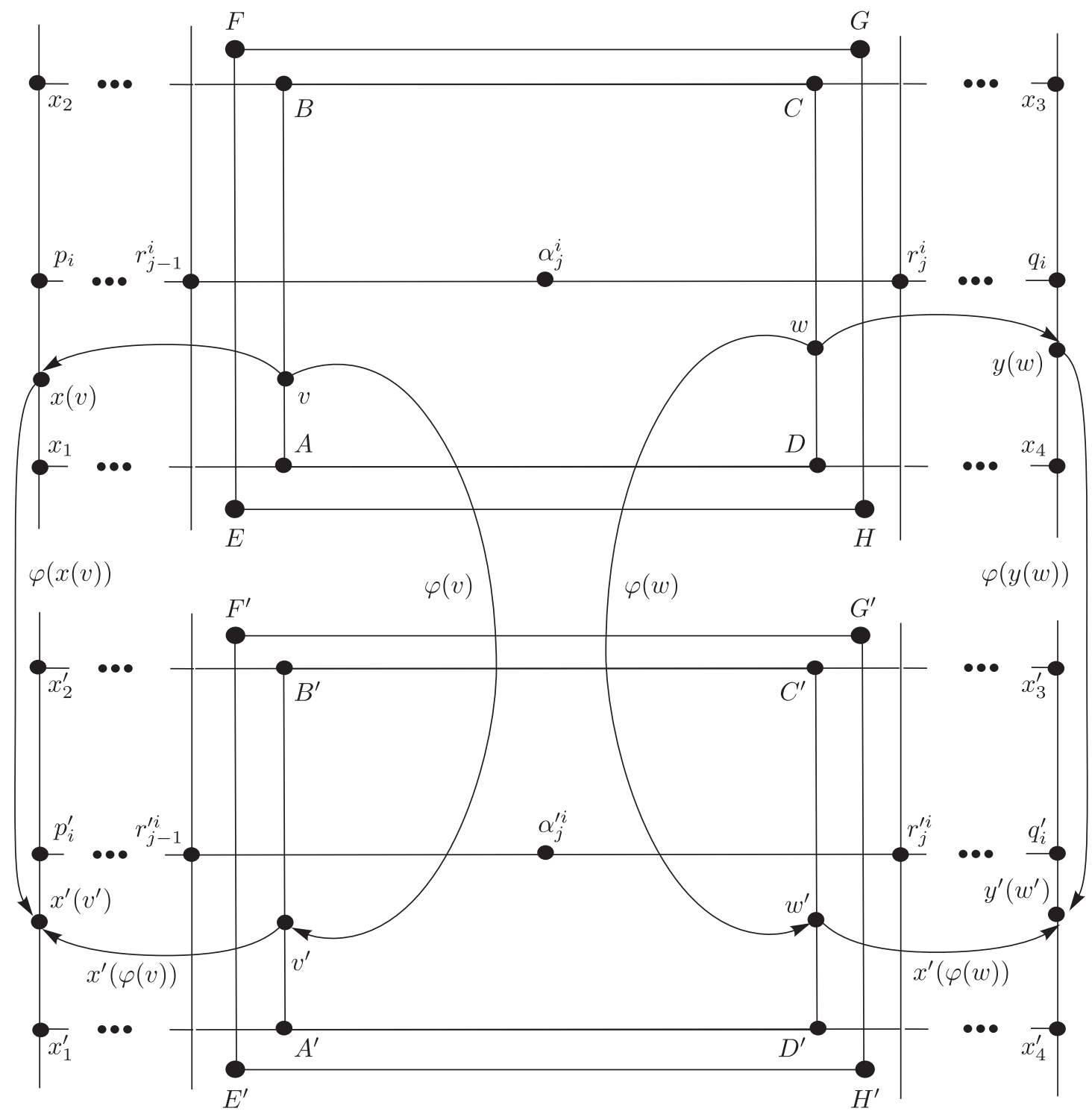

Fig. 7. Conjugation on $A B$ and $C D$. 
Consider the quadrilaterals $A E F B, B F G C, C G H D, D H E A$ obtained by dividing the annulus $K_{j}^{i}$ into segments $A E, B F, C G, D H$. We foliate each of them by segments of stable manifolds $W_{x}^{s}$. The set of the segments thus obtained is denoted by $\mathcal{W}$. Then we foliate each of the quadrilaterals by segments that are transversal to the segments from $\mathcal{W}$, the set of which we denote by $\mathcal{R}$ (see Fig. 8).

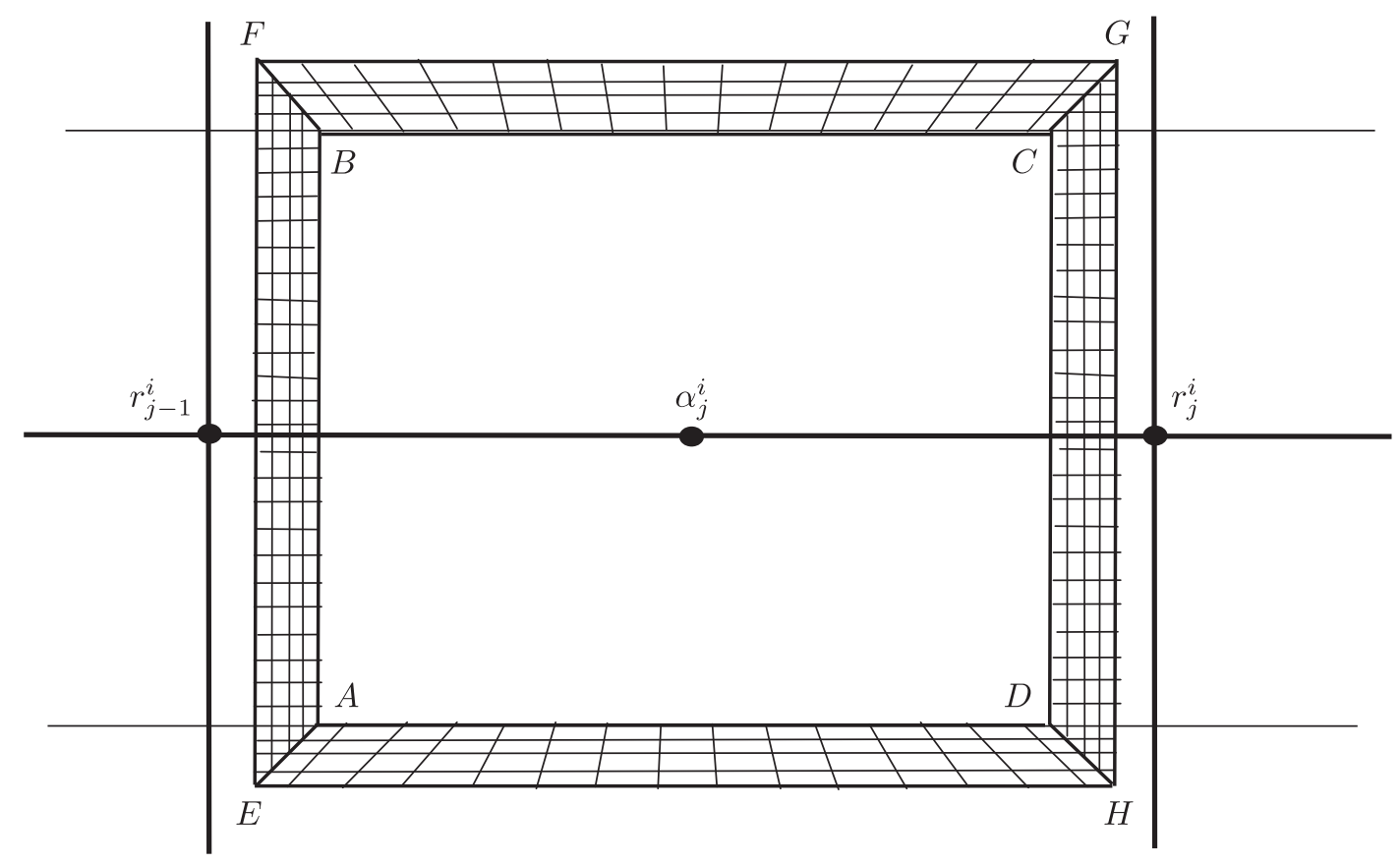

Fig. 8. Foliation on the fundamental domain $K_{j}^{i}$.

Consider an annulus $K_{j}^{\prime i}$ that is similarly divided into four quadrilaterals. We foliate each quadrilateral of this annulus by segments as follows: if there is a segment from the set $\mathcal{R}$ with boundary points $x$ and $y$, then there is a segment from the set $\mathcal{R}^{\prime}$ with boundary points $x^{\prime}=\varphi_{\partial}(x), y^{\prime}=\varphi_{\partial}(y)$. Then on each quadrilateral of the annulus $K_{j}^{i}$ homeomorphism $\varphi_{\partial}$ induces a map $\varphi_{\mathcal{W}}: \mathcal{W} \rightarrow \mathcal{W}^{\prime}, \varphi_{\mathcal{R}}: \mathcal{R} \rightarrow \mathcal{R}^{\prime}$. Then a homeomorphism $\varphi_{K_{j}}$ of the annulus $K_{j}=A B C D E F G H$ is defined by the following rule: if $z$ is the intersection point of segments $\mathbf{w} \in \mathcal{W}$ and $\mathbf{r} \in \mathcal{R}$, then $\varphi_{K_{j}}(z)$ is the intersection point of segments $\varphi_{\mathcal{W}}(\mathbf{W})$ and $\varphi_{\mathcal{R}}(\mathbf{r})$. The equality $\left.\varphi_{W^{u}\left(\alpha_{j}^{i}\right)}=\phi^{-k}\left(\varphi_{K_{j}} \phi^{k}(z)\right)\right)$, where $z \in K_{j}, k \in \mathbb{Z}$, defines the required homeomorphism $\varphi_{W^{u}\left(\alpha_{j}^{i}\right)}: W^{u}\left(\alpha_{j}^{i}\right) \rightarrow W^{u}\left(\alpha_{j}^{i}\right)$.

\section{Conflict of Interest}

The authors declare that they have no conflict of interest.

\section{References}

[1] Franks, J., Anosov Diffeomorphisms, in Global Analysis: Proc. Sympos. Pure Math. (Berkeley, Calif., 1968): Vol.14, Providence, R.I.: AMS, 1970, pp. 61-93.

[2] Grines, V.Z., The Topological Equivalence of One-Dimensional Basic Sets of Diffeomorphisms on Two-Dimensional Manifolds, Uspekhi Mat. Nauk, 1974, vol. 29, no. 6(180), pp. 163-164 (Russian). 
[3] Grines, V.Z., The Topological Conjugacy of Diffeomorphisms of a Two-Dimensional Manifold on One-Dimensional Orientable Basic Sets: 1, Trudy Moskov. Mat. Obsc., 1975, vol. 32, pp. 35-60 (Russian).

[4] Grines, V.Z., The Topological Conjugacy of Diffeomorphisms of a Two-Dimensional Manifold on One-Dimensional Orientable Basic Sets: 2, Trudy Moskov. Mat. Obsc., 1977, vol. 34, pp. 243252 (Russian).

[5] Grines, V., Medvedev, T., and Pochinka, O., Dynamical Systems on 2- and 3-Manifolds, Dev. Math., vol. 46, New York: Springer, 2016.

[6] Grines, V.Z. and Zhuzhoma, E. V., Structurally Stable Diffeomorphisms with Basic Sets of Codimension One, Izv. Math., 2002, vol.66, no. 2, pp. 223-284; see also: Izv. Ross. Akad. Nauk Ser. Mat., 2002, vol. 66, no. 2, pp. 3-66.

[7] Mañé, R., A Proof of the $C^{1}$ Stability Conjecture, Publ. Math. Inst. Hautes Etudes Sci., 1987, vol. 66, pp. 161-210.

[8] Plykin, R. V., The Topology of Basic Sets of Smale Diffeomorphisms, Sb. Math., 1971, vol. 13, no. 2, pp. 297-307; see also: Mat. Sb. (N.S.), 1971, vol. 84(126), no. 2, pp. 301-312.

[9] Robinson, C., Structural Stability of $C^{1}$ Diffeomorphisms, J. Differential Equations, 1976, vol. 22, no. 1, pp. 28-73.

[10] Zhuzhoma, E. V. and Medvedev, V.S., On Nonorientable Two-Dimensional Basic Sets on 3-Manifolds, Sb. Math., 2002, vol.193, nos.5-6, pp.869-888; see also: Mat. Sb., 2002, vol.193, no.6, pp. $83-104$. 